\title{
ELECTROFORÉSIS: FUNDAMENTOS, AVANCES Y APLICACIONES
}

\author{
Electrophoresis: fundamentals, advances and applications
}

EPISTEMUS

ISSN: 2007-8196 (electrónico)

ISSN: 2007-4530 (impresa)

Montalvo Navarro Carlos Antonio ${ }^{1}$

Lugo Flores Marco Antonio ${ }^{2}$

Recibido: 20 de septiembre de 2016,

Aceptado: 30 de noviembre de 2016

Autor de Correspondencia:

Lic. Montalvo Navarro Carlos Antonio

Correo:carmona1994@gmail.com

\section{Resumen}

La electroforésis es una técnica para separación de biomoléculas según su movilidad y naturaleza (generalmente ácidos nucleicos o proteínas) en un campo eléctrico sobre una matríz porosa, cuya composición depende de la biomolécula a analizar. Para la separación de ácidos nucléicos se utilizan matrices de agarosa y para la separación de proteínas se utilizan matrices de poliacrilamida. Esta técnica representa una herramienta fundamental de análisis cuantitativos en diversos campos de ciencias biológicas como biología molecular, bioquímica o proteómica. Entre las distintas plataformas de electroforésis, las más utilizadas en análisis de ácidos nucléicos son la electroforésis en gel de agarosa, la electroforésis de campo pulsado (PFGE) o la electroforésis en gel con gradiente de desnaturalización (DGGE), y las más utilizadas para análisis de proteínas son la electroforésis en gel de poliacrilamida con duodecil sulfato de sodio (SDS) y la electroforésis bidimensional. Esta revisión discutirá las técnicas previamente mencionadas y sus recientes aplicaciones.

Palabras clave: electroforésis, ADN, PAGE, PFGE, DGGE

\section{Abstract}

Electrophoresis is a technique for separating biomolecules according to their mobility and nature (usually nucleic acids or proteins) in an electric field on a porous matrix, whose composition depends on the biomolecule to be analyzed. For the separation of nucleic acids, agarose matrices are used and for the separation of proteins, polyacrylamide matrices are used. This technique represents a fundamental tool of quantitative analysis in various fields of biological sciences such as molecular, biochemical or proteomic biology. Among the different electrophoresis platforms, the most used in nucleic acid analysis are agarose gel electrophoresis, pulsed field electrophoresis (PFGE) or denaturing gradient gel electrophoresis (DGGE), and the most used for analysis of proteins are polyacrylamide gel electrophoresis with sodium duodecyl sulfate (SDS) and two-dimensional electrophoresis. This review will discuss the previously mentioned techniques and their recent applications.

Keywords: electrophoresis, DNA, PAGE, PFGE, DGGE.

1 Universidad autónoma de baja california: campus ensenada Correo: carmona1994@gmail.com

2 Instituto tecnológico de los Mochis Correo: marco_lugo029@hotmail.com 


\section{INTRODUCCIÓN}

Los microrganismos son un grupo de seres vivos que se encuentran en una gran variedad de lugares como lo son las plantas, animales y humanos en donde coexisten con el huésped ayudando a realizar un $\sin$ fin de funciones. Sin embargo, algunos de estos como los microorganismos patógenos pueden ocasionar enfermedades en los organismos mencionados provocando la muerte de los mismos. Desde la perspectiva de la salud, en seres humanos es necesario establecer protocolos que permitan la identificación de estos agentes perjudiciales y prevenir el efecto patógeno que generan sobre las personas. El estudio del ADN de los microorganismos ha potenciado la identificación de estos a partir de diferentes técnicas moleculares, tal es caso de la electroforésis, una técnica que permite el procesamiento de los fragmentos de ADN que al ser regiones muy conservadas dentro de cada especie, es posible identificar a estos. El presente estudio proporciona información acerca del fundamento de la técnica de electroforésis, los procedimientos y reactivos requeridos para la misma, así como los avances y nuevas técnicas que han surgido con el paso del tiempo.

\section{DESARROLLO}

\section{Métodos de separación de ácidos nucléicos.}

Electroforésis en gel de agarosa. La creación de la electroforésis como un método de separación de ADN bicatenario de cadenas simples se remonta al año 1962, elaborado por Matsubara y Takagi, donde se utilizaba al almidón como gel de corrimiento. La necesidad de analizar moléculas de ADN de longitudes variables propició el estudio de otros compuestos que pudieran utilizarse como gel de corrimiento en las pruebas de electroforésis, surgiendo la agarosa en 1969 como un candidato adecuado debido a las características que posee. La agarosa es un polisacárido obtenido del aislado de agar de algas rojas marinas, el cual está constituido por unidades repetidas de agarobiosa, sustituida de manera extensa en grupo ester de sulfato, acido pirúvico cetal y esteres metílicos. Dicho polímero es de interés en el análisis del ADN debido a las características que posee al ser gelificado, consiste en una bobina aleatoria estructurada por una doble hélice en etapas iniciales de la gelificación y a medida que avanza la gelificación se forman las dobles hélices finales. La configuración mencionada forma una red fibrosa que puede ser utilizada como filtro para la clasificación de diferentes moléculas, donde la concentración de agarosa es inversamente proporcional al tamaño del poro. Ha sido reportado un límite de tamaño máximo de fragmentos de ADN que pueden ser analizados con gel de agarosa, siendo entre 100 a 2000 pb a una concentración de 2-3\% del polisacárido. Por otro lado la longitud mínima del fragmento se establece a valores superiores a $50 \mathrm{~kb}$, donde la concentración recomendada es de $0.25 \%$ de agarosa [15]. La aplicación de la electroforésis con geles de agarosa se ha implementado una gran variedad de estudios como lo es el ensayo de comentado esencial (ensayo de una sola célula). Dicha técnica es utilizada para determinar el daño o reparación del ADN en células eucariotas y tejidos disgregados a partir de la adición de la suspensión de células junto con la agarosa sobre el molde para formar el gel delgado. Después se realiza la lisis de las células con un detergente (Triton X-100) y $\mathrm{NaCl} 2 \mathrm{M}$ para la eliminación de histonas del ADN. Las moléculas que permanecen adheridas al gel reciben el nombre de nucleoides que se caracterizan por mantener el superenrollamiento del ADN. De acuerdo al modelo propuesto por Cook y col. En 1970, estipula que las moléculas de ADN están unidas a una matriz nuclear formando una secuencia donde cada unidad estructural genera un bucle. Entonces, al ocasionar rupturas de la cadena de ADN se dice que el superenrrollamiento se relaja y al ser aplicado un campo electroforético, dicho bucle puede migrar hacia el anodo de la cámara de electroforésis. A mayor cantidad de rupturas se presenta una mayor cantidad de $A D N$ en la cola del cometa, que después de teñirse (4,6-diamidino-2fenilindol), la intensidad relativa de la fluorescencia de la cola se mide como índice de frecuencia de ruptura del ADN por medio de microscopia de fluorescencia.

Aplicaciones. La aplicación de dicho ensayo abarca el estudio de células sanguíneas provenientes de animales o humanos, células de hemolinfa de moluscos e insectos, esperma, tejidos animales disgregados, levaduras, núcleos liberados de tejidos vegetales, en otras palabras es aplicable a cualquier tipo de célula eucariota que pueda obtenerse como una célula única [12].

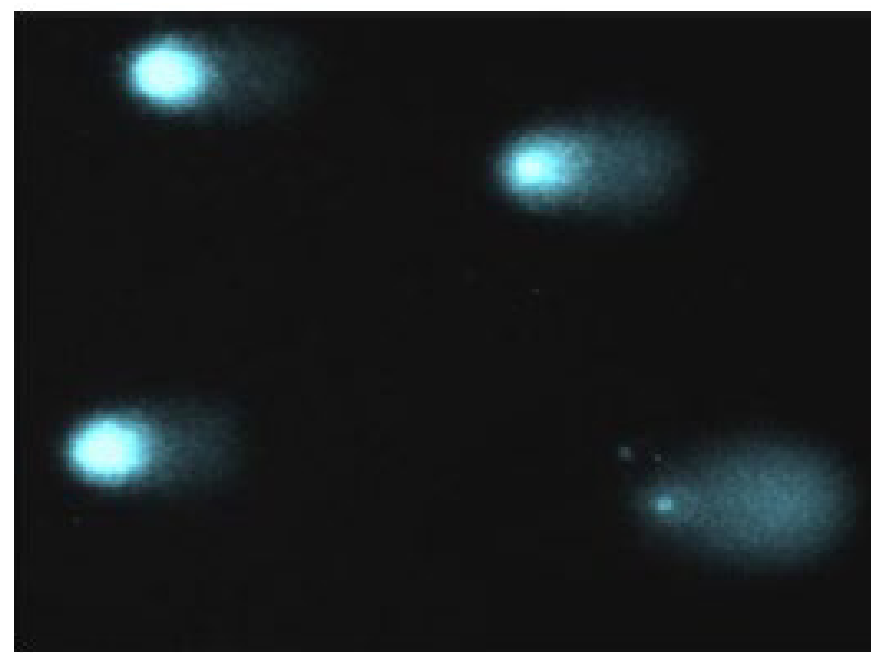

Figura 1. Imágenes de ensayo de cometa obtenidas por microscopia de fluorescencia

Electroforésis de campo pulsado (PFGE). El desarrollo y modificaciones de la electroforésis convencional permiten generar nuevas metodologías para mejorar el análisis e identificación de contenido genético de células eucariotas y procariotas. Scwartz y Cantor demostraron que la implementación de campos eléctricos podía ser utilizada 
químicos (generalmente urea o formamida) a través de los cuales el DNA migra por electroforésis. A medida que el DNA migra por el gradiente, cada molécula empezara a desnaturalizarse a una concentración particular del desnaturalizante dependiendo del \%GC en la molécula y el orden de las bases en la secuencia. La migración de las moléculas se retardada cuando sucede la desnaturalización. La técnica DGGE se representa esquemáticamente en la Fig. 3.

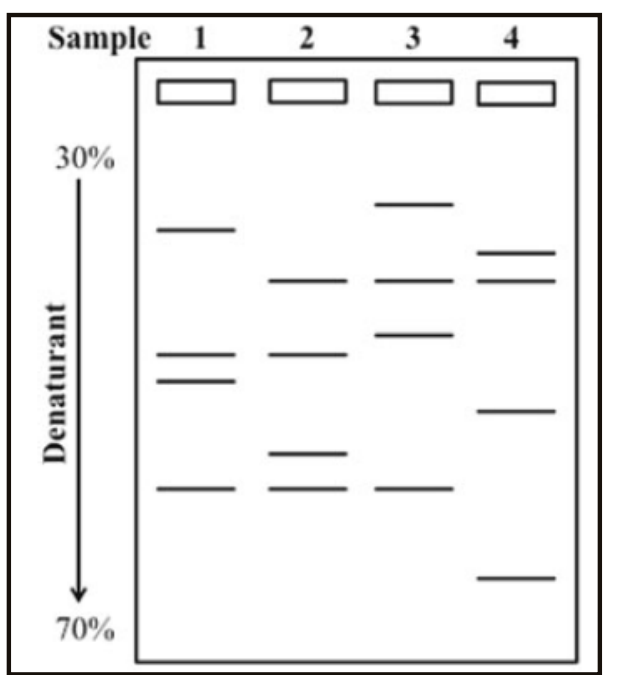

Figura 3. Diagrama esquemático de un DGGE

Un gradiente de temperatura en lugar de un gradiente químico desnaturalizante puede ser utilizado, en este caso la técnica es Ilamada Electroforésis en Gel con Gradiente de Temperatura (TGGE). Recientemente, DGGE se ha convertido en una herramienta importante en la ecología microbiana, donde ha sido utilizado como método de huella molecular para evaluar la diversidad microbiana en comunidades con mezclas complejas de microorganimos.

Las investigaciones realizadas por Subasinghe et al., [10] para la identificación de bacterias en productos microbianos utilizaron la técnica de DGGE, y con ella encontraron que la formación de múltiples bandas en el gel representaban a una misma bacteria en lugar de un consorcio bacteriano. Esto fue atribuido a inconvenientes con el PCR elaborado previamente. Otro ejemplo de aplicación del DGGE fue el realizado por Xin et al., [11] en el análisis de comunidades bacterianas asociadas con pericoronitis asintomática y sintomática, una enfermedad bucal inflamatoria. El resultado del perfil bacteriano en pacientes clínicos mostro una menor diversidad de microorganismos que en los pacientes control (sin enfermedad), este descubrimiento sugirió que la microbiota asociada a pericoronitis se vuelve menos diversa, quizá debido a que ciertos grupos de microorganismos dominan el las biopelículas a medida que la pericoronitis progresa.

\section{Métodos de separación de proteínas.}

Electroforésis en gel de poliacrilamida (PAGE). La electroforésis es una técnica para la separación de moléculas según la movilidad de éstas en un campo eléctrico. Los geles de poliacrilamida son geles químicamente entrelazados formados por la reacción de acrilamida con un agente de entrecuramiento bifuncional como el Bis [8]. La electroforésis con geles de poliacrilamida (PAGE) es útil para separar moléculas por tamaño-carga y hay muchos sistemas diferentes dependiendo de la muestra y

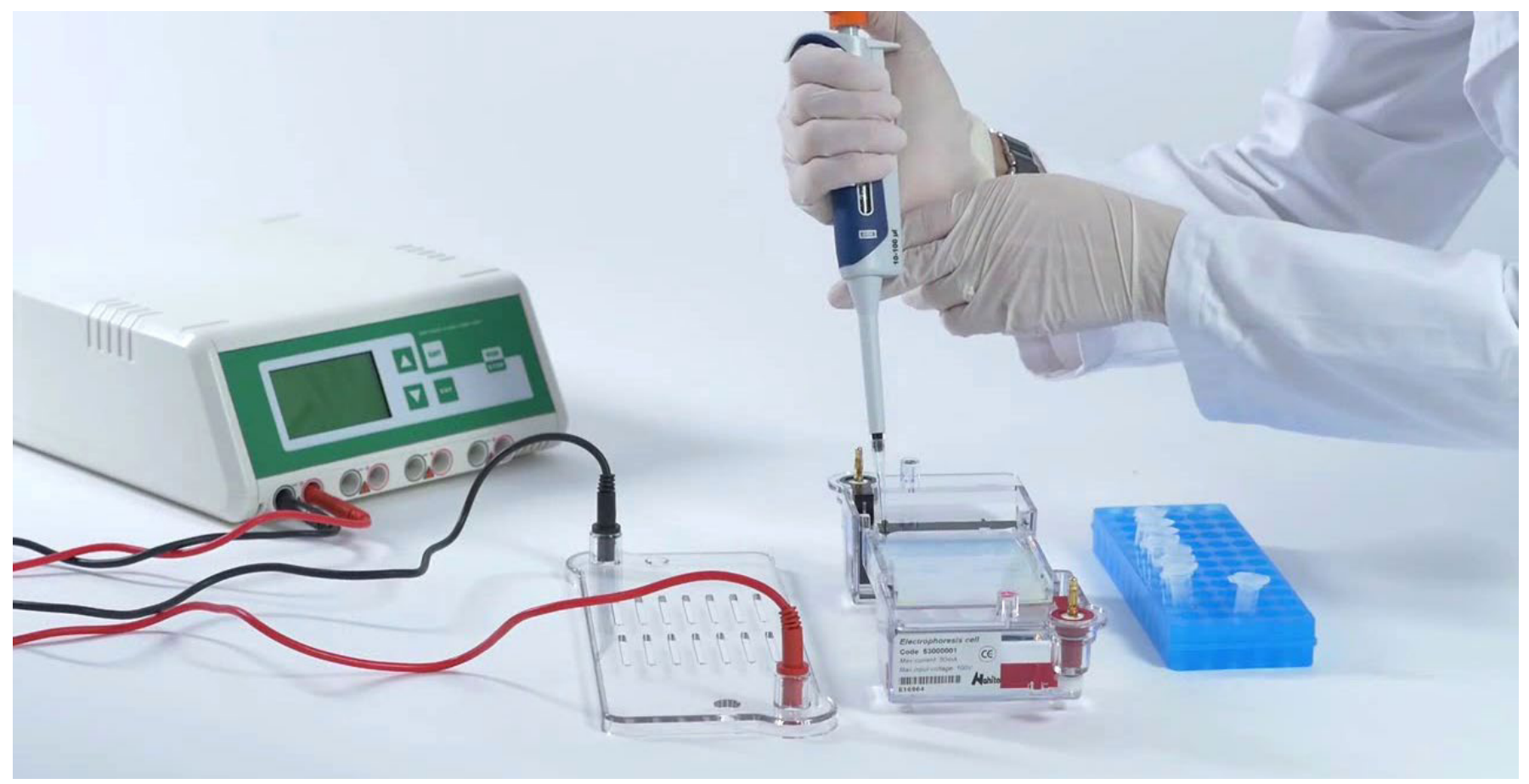


sus posteriores aplicaciones [2]. Las propiedades que debe tener un medio de soporte ideal son [6]:

- Naturaleza química inerte (no debe reaccionar con el analito o con cualquier reactivo utilizado)

- Alta conductividad eléctrica

- No debe absorber el analito

- Porosidad controlada para lograr un efecto de separación deseado

- Transparencia para lograr una tinción e imagen exitosa

- Baja toxicidad

- Fácil disponibilidad de los reactivos utilizados

- Rigidez razonable para un fácil manejo de la matriz

- Electroendoosmosis: flujo de un buffer a través de una superficie cargada

Los geles de poliacrilamida tienen todas las propiedades descritas anteriormente.

Formatos de PAGE. Varios formatos y versiones de PAGE han sido desarrolladas, como:

1.SDS-PAGE

2.Gradiente de SDS-PAGE

3.PAGE de Ácido acético - Urea para proteínas básicas

4. Gel de electroforésis discontinua con Bromuro de cetiltrimetilamonio

5. Electroforésis con enfoque isoeléctrico

6. Electroforésis capilar

7. Electroforésis bidimensional

La popularidad de los geles de poliacrilamida proviene de varias propiedades fundamentales como claridad óptica, neutralidad eléctrica y disponibilidad en un amplio rango de porosidad. Su fórmula química, como comúnmente se polimeriza a partir de acrilamida y N, NOmetileno bisacrilamida (Bis), se muestra en la Fig. 4, junto con la de los dos catalizadores más ampliamente usados, el peroxodisulfato (amonio o potasio) y N, N, NO, NOtetrametiletilendiamina (TEMED) [6].

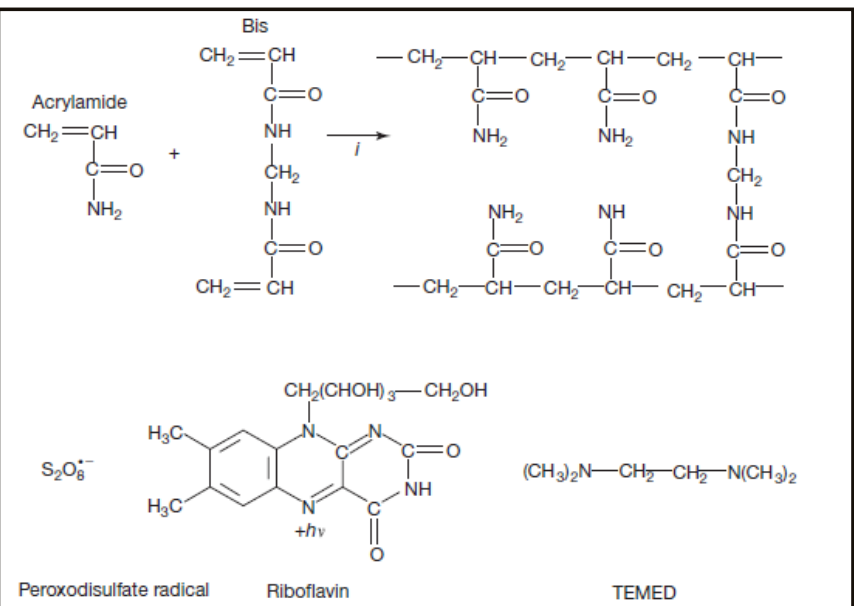

Figura. 4 Reacción de polimerización de la acrilamida e iniciadores de la polimerización.

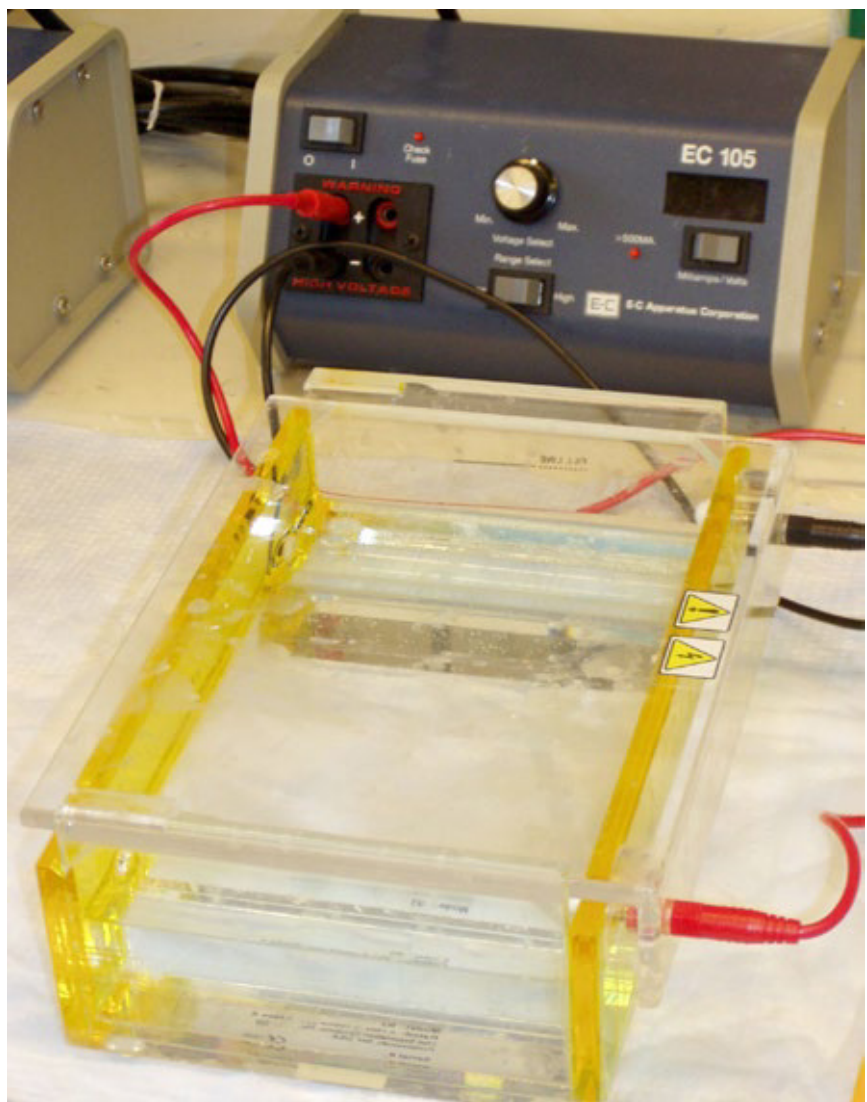

Electroforésis en gel de poliacrilamida con duodecil sulfato de sodio (SDS-PAGE). SDS es un detergente que desnaturaliza estructuras secundarias y estructuras terciarias no enlazadas con disulfuro y las reviste con una carga negativa que se correlaciona con su longitud, permitiendo que los pesos moleculares sean estimados. La movilidad a través del gel puede verse afectada por el estado de la proteína (por ejemplo, fosforilación y presencia de moléculas multiméricas) [2]. La electroforésis SDS fue el siguiente paso lógico después de la electroforésis discontinua. Mientras que esta última discrimina las macromoléculas en función del tamaño y la carga superficial, la electroforésis SDS fracciona las cadenas polipeptídicas esencialmente en función de su tamaño. Por lo tanto, es un método simple, pero poderoso y confiable para la determinación de la masa molecular.

La muestra de proteína $(1 \mathrm{mg} / \mathrm{mL})$ generalmente se desnaturaliza en $100 \mathrm{mmol} / \mathrm{L}$ fosfato $\mathrm{pH}$ 7.0, que contiene $1 \%$ SDS, 1\% 2-mercaptoetanol, 5\% -10\% de sacarosa (para aumentar la densidad de la muestra para cargar el gel) y trazas de bromofenol azul (como un colorante de rastreo para monitorear la formación de límites en sistemas discontinuos y para verificar la terminación de la corrida). Después calentando a $100^{\circ} \mathrm{C}$ durante 5 minutos, la muestra se deposita generalmente en posillos en la placa de gel [6].

Para detectar las bandas se realiza una tinción con varios reagentes. Después de la tinción, las bandas pueden ser cuantificadas por densitometría. Adicionalmente a la 


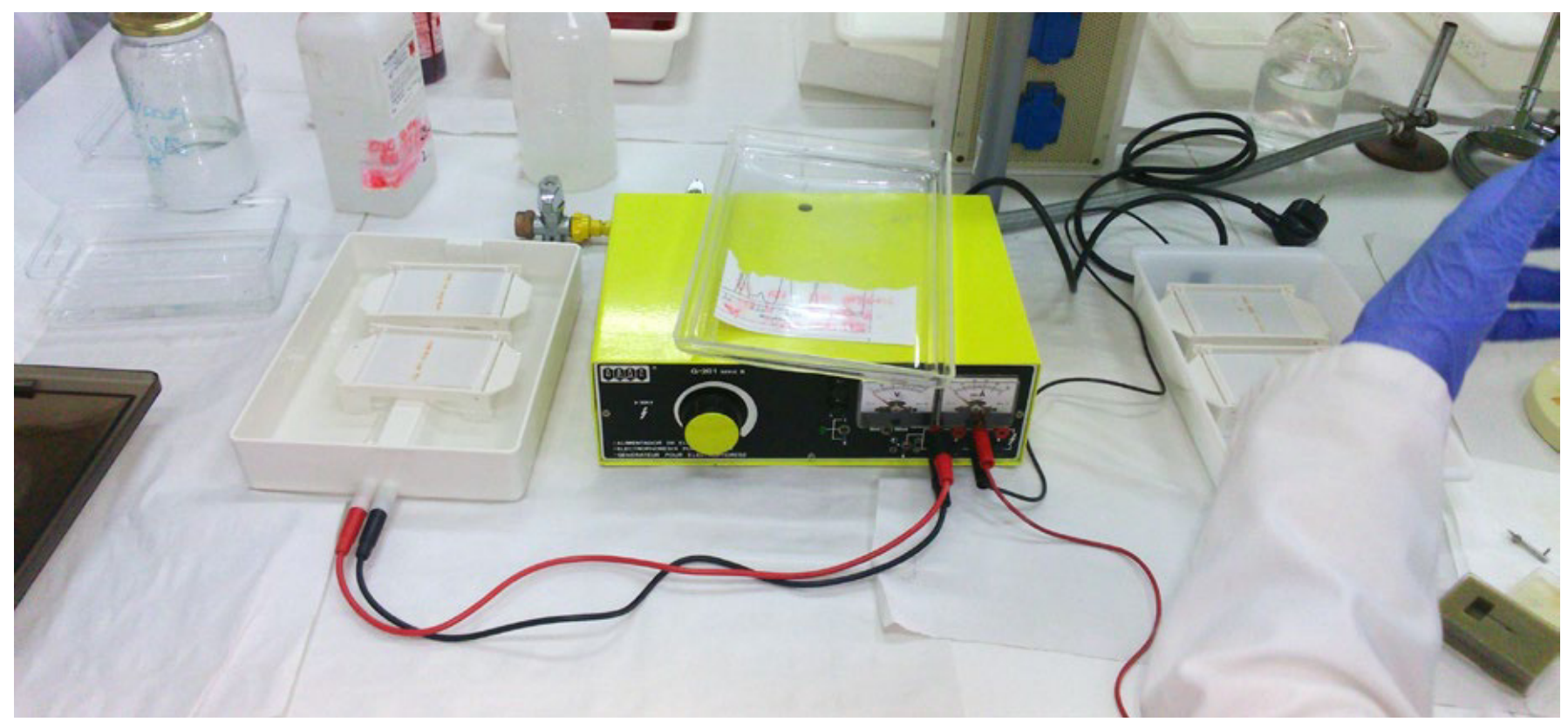

transcriptómica, proteómica, metagenómica) ya que estas técnicas se han combinado con otras plataformas como $\mathrm{PCR}$, técnicas de secuenciación de nueva generación y espectroscopía de masas acoplada a cromatografía de líquidos, entre otras lo cual ha permitido una mejor resolución y precisión de resultados experimentales. Sin embargo, las limitaciones asociadas a esta técnica deben solucionarse para poder aproximarnos a un mejor entendimiento de la composición, funcionamiento y cambios en el tiempo de los sistemas biológicos.

\section{BIBLIOGRAFÍA}

[1] Kucharska, E., \& Wróblewska, B. Food allergens. In Toxins and Other Harmful Compounds in Foods, 2017

[2] Brunelle, J. L., \& Green, R. One-dimensional SDSpolyacrylamide gel electrophoresis (1D SDS-PAGE). In Methods in Enzymology (1st ed., Vol. 541), 2014

[3] Lee, M. K., Kim, J. K., \& Lee, S. Y. Effects of fermentation on SDS-PAGE patterns, total peptide, isoflavone contents and antioxidant activity of freeze-thawed tofu fermented with Bacillus subtilis. Food Chemistry, 249(July 2017), 60-65, 2018

[4] Muharram, M. M., \& Abdel-Kader, M. S. Utilization of gel electrophoreses for the quantitative estimation of digestive enzyme papain. Saudi Pharmaceutical Journal, 25(3), 359364, 2017

[5] Rabilloud, T., \& Lelong, C. Two-dimensional gel electrophoresis in proteomics: A tutorial. Journal of Proteomics, 74(10), 1829-1841, 2011

[6] Righetti, P. G. ELECTROPHORESIS | Polyacrylamide Gels. In Encyclopedia of Analytical Science (3rd ed.), 2005a

[7] Righetti, P. G. ELECTROPHORESIS | Two-Dimensional Gels. In Encyclopedia of Analytical Science (3rd ed.), 2005b

[8] Stellwagen, N. C. Electrophoresis of DNA in agarose gels, polyacrylamide gels and in free solution. Electrophoresis, 30(SUPPL. 1), 188-195, 2009

[9] Strathdee, F., \& Free, A. (n.d.). Chapter 9 Denaturing Gradient Gel Electrophoresis ( DGGE ). 1054, 145-157, 2013

[10] Subasinghe, R. M., Samarajeewa, A. D., Scroggins, R., \& Beaudette, L. A. Evaluation of denaturing gradient gel electrophoresis ( DGGE) and next generation sequencing (
NGS ) in combination with enrichment culture techniques to identify bacteria in commercial microbial-based products. Journal of Microbiological Methods, 161(April), 118-130, 2019

[11] Zhang, X., Sun, Z., \& Yang, Q. Application of Denaturing Gradient Gel Electrophoresis to the Analysis of Bacterial Communities Associated With Asymptomatic and Symptomatic Pericoronitis. Journal of Oral Maxillofacial Surgery, 76(3), 483-489, 2017

[12] Azqueta, A., and Collins, A. R. "The essential comet assay: a comprehensive guide to measuring DNA damage and repair," Archives of toxicology, vol. 87, no. 6, pp. 949-968, 2013.

[13] Lopez-Canovas, L., Benitez, M. B. M., Isidron, J. A. H., \& Soto, E. F."Pulsed Field Gel Electrophoresis: Past, present, and future," Analytical biochemistry, submitted for publication, 2019.

[14] Pulpipat, T., Lin, K. H., Chen, Y. H., Wang, P. C., \& Chen, S. C. "Molecular characterization and pathogenicity of Francisella noatunensis subsp. orientalis isolated from cultured tilapia (Oreochromis sp.) in Taiwan," Journal of fish diseases, vol. 42, pp. 643-655, 2019.

[15] Upcroft, P., \& Upcroft, J. A. "Comparison of properties of agarose for electrophoresis of DNA," Journal of Chromatography B: Biomedical Sciences and Applications, vol. 618 no. (1-2), pp. 79-93, 1993.

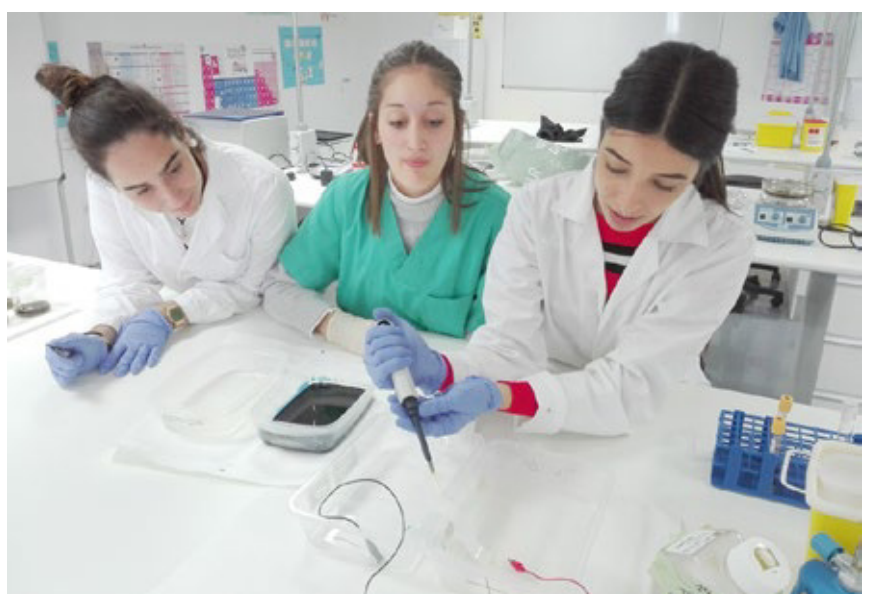

